\title{
Semantic Representation of Process and Service Compliance - A Case Study in Emergency Planning
}

\author{
Aygul Gabdulkhakova ${ }^{1}$, Birgitta König-Ries ${ }^{1}$, and Norris Syed Abdullah ${ }^{2}$ \\ ${ }^{1}$ Friedrich-Schiller-University Jena, Germany \\ \{aygul .gabdulkhakova, birgitta.koenig-ries\} @uni-jena.de \\ ${ }^{2}$ The University of Queensland, Brisbane, Australia \\ norriseitee.uq.edu.au
}

\begin{abstract}
Emergency events like natural disasters and large scale accidents pose a number of challenges to handle. The requirement to coordinate a wide range of organizations and activities, public and private, to provide efficient help for the victims can often be complicated by the need to comply with requisite policies and procedures. Current process and service models that represent domains such as emergency planning do not provide sufficient artefacts with respect to compliance requirements. In this paper, we argue that techniques for compliance management in business processes can be applied to the emergency domain. We provide a high level model for the representation of compliance requirements within business processes and services. Hence, we demonstrate the application of the model in the emergency planning domain. Finally, we present an analysis derived from the case study that identifies the current limitations and requirements for semantic extensions of process and service models in order to cater for compliance.
\end{abstract}

Keywords: emergency management, SOA, semantic web services, and compliance management.

\section{Introduction}

The organizations involved in mass casualty incidents (MCI), i.e., police, medical services, and fire and rescue forces, are faced with manifold challenges. A mass casualty incident (MCI) is a special incident in which the personnel and equipment of the rescue forces at the scene are overwhelmed by the number and severity of casualties at that incident. The main problem for the operations manager in such situations is to manage the gap between actually available resources and necessary resources [1]. Typically, this assignment needs to be done on-the-fly once the disaster has occurred: While for some disasters (e.g., a fire in a football stadium), rather detailed emergency plans can be developed beforehand, there are also numerous disasters where this is not the case. Consider, e.g., hurricanes or traffic accidents. These disasters can occur anywhere and can vary widely in severity. Depending on the exact location, access to the accident site will differ, the number, experience, and equipment of available emergency personnel and volunteers will also depend on the location. Often, only 
limited information about the incident will be available in the beginning. More information about the situation will become known over time, only.

Thus, a system is needed that can efficiently assign emergency personnel and equipment to the necessary tasks according to their abilities and organizational structures (police, medical services, fire and rescue services). The SpeedUp project [www.speedup.uni-jena.de] aims to develop a framework that provides IT-support for the cooperation and communication of emergency management organizations. Up to now, such cooperation is achieved more or less manually by orally exchanging information or exchanging print outs. In the case of scenarios such as mass casualty incidents, however, personal consultations or the procurement and dispatch of documents are virtually impossible to manage. Processes are inefficient and precious time is lost as a result: factors that, in the worst case, raise the number of victims. In our ongoing work, we are investigating the possibility to dynamically map emergency management processes to varying underlying resources using semantic web service technology. The aim is to (semi-)automatically compose processes using the available resources.

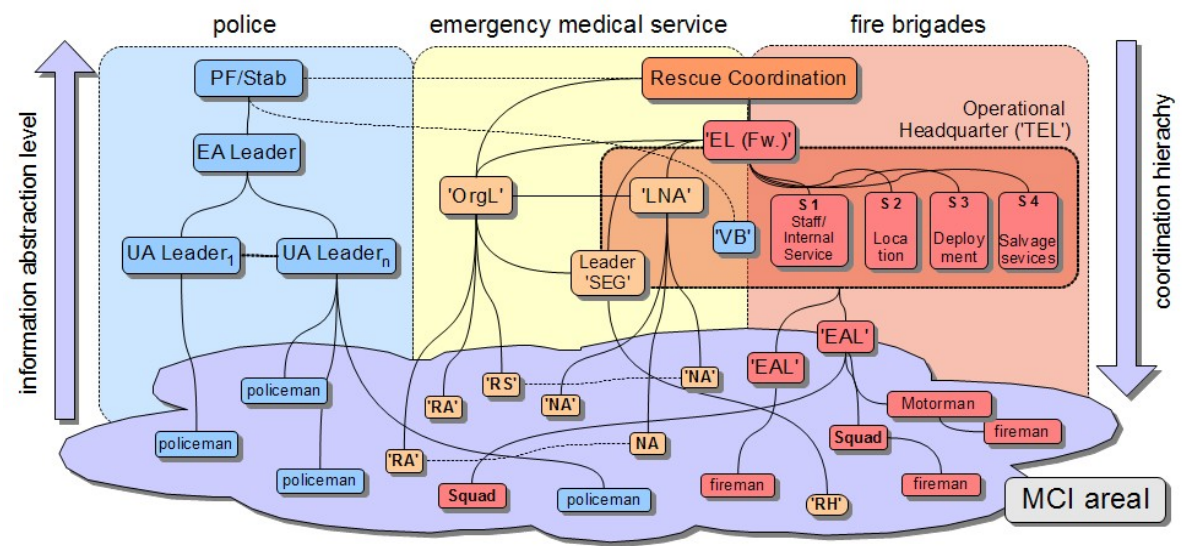

EA Leader - leader of an operational area; UA Leader - subsection leader; EL - local operation manager; $S$ I- S4 functional areas; $\operatorname{Org} L$ - Organizational Medical Leader; $L N A$ - Leading Emergency Doctor; $R H$ - Emergency Medical Technician; $R A$ - Paramedic; $R S$ - Emergency Medical Technician; NA - Emergency Doctor; EAL leader of an operational area; VB - liaison officer; PF (Polizeifuehrer)/Stab - commander of police/ Crisis Management Group (CMG).

Fig. 1. Core roles within organizations in the MCI scenario

It is important, however, that these processes and the assigned resources do not only meet functional requirements, but also obey the rules and standards governing emergency organizations. Consider an accident on a motorway with a number of injured people. In handling this situation, at least fire fighters, first aid personnel, and police will be involved and special management structures will be established between them. For example, the police use a Special Organisational Structure, while the fire and emergency medical services establish a Technical Operation Management respectively OrgL (organisational medical leader) and LNA (leading emergency doctor) (see Figure 1). Different roles in this structure are assigned with different rights based on legal requirements or organizational rules. A special characteristic in 
such a scenario is that the operation managers should handle tasks whose goals arise and can quickly change during the mission itself [2]. So, the basic idea is to develop a mechanism that supports operation managers in achieving those goals by (semi)automatically assigning or suggesting appropriate resources and at the same time ensures that existing rules, such as access restrictions on a particular personal data, commands are given by authorized personnel etc., are obeyed by the dynamically created processes. In this paper, we propose such a system based on semantic web service technology. Resources are modeled as services and will be discovered and bound at run time. To achieve this, services need to be described in a machineprocessable manner. In addition, we need to make sure that only services that meet rules and regulations are used within a process. Here, we argue that techniques for compliance management known from BPM can be adapted to our domain. In the remainder of the paper, we discuss how this can be achieved. First, we introduce the semantic service descriptions we use. Second, we look at compliance requirements in our domain and discuss how they could be formalized. Finally, we present our architecture that brings together the different building blocks. Thereafter, we will give an overview of the related work and conclude.

\section{Semantic Service Descriptions}

We have decided to base our system on a service oriented architecture with semantic, i.e., machine-processable service descriptions, as this is an ideal architecture to support flexibility and robustness in dynamic situations. These descriptions enable an automatic assignment of suitable service offers to service requests. In case a service is no longer available, performs badly or inadequate in a certain situation, another service offer can be chosen without affecting the overall behavior.

To describe services semantically, we use the DIANE Service Description language (DSD) and its matchmaker [3] to provide such descriptions and to automatically match service requests and offers in order to find the most appropriate pairing for both. The language is not based on one of the existing ontology or logic languages such as the often used description logic or f-logic, but uses an ontology language and a reasoning mechanism that is specialized for service discovery: the DIANE Elements (DE). Service offers are described as sets of required preconditions and achieved effects. By the introduction of variables, DSD allows to seamlessly integrate the descriptions of which functionality is provided and the form of message flow, viz., which inputs a service expects and which outputs it is able to produce. DSD descriptions of both offers and requests are basically directed graphs. The matchmaker traverses the request graph and tries to find and configure matching offers by implementing a subset test. This powerful and efficient matchmaker is at the heart of the accompanying semantic service middleware that supports the whole process of service matching, selection, and automatic invocation [3].

Figure 2 illustrates an example of a service offer that allows transmitting a text message (e.g., information about number of affected persons) from the leading emergency doctor to the organizational medical leader. The description tells that the effect of the service execution will be that a text message (TextMessage is an input 


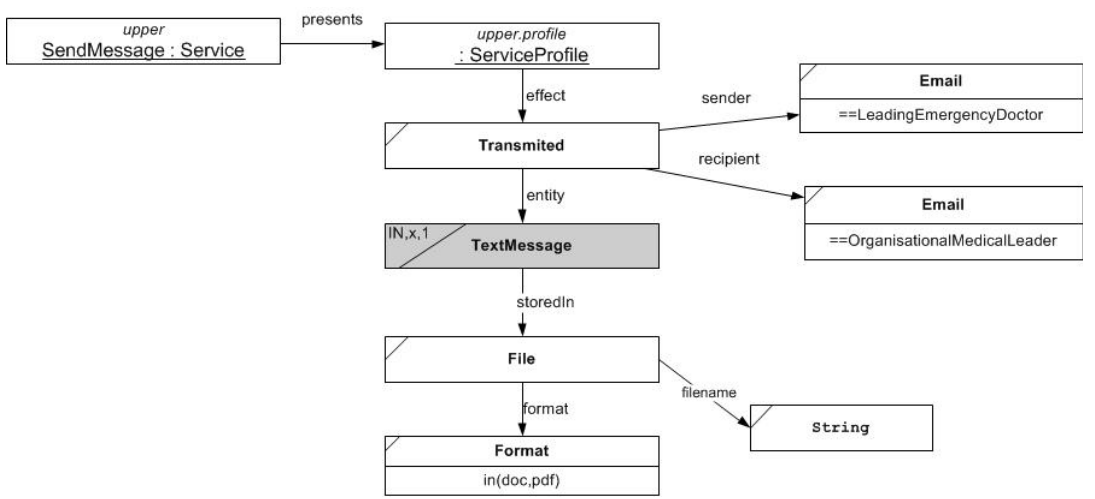

Fig. 2. Service Offer "SendMessage"

parameter of the service) will be sent from the leading emergency doctor to the organizational medical leader. This message needs to be in .doc or .pdf format and the filename needs to be provided as an input. Service requests are formulated similarly and are then automatically matched with all available service offers to find the best matching currently available service.

We envision emergency personnel to have access to a tool that will trigger service requests at certain steps in emergency management process. For instance, in the event that leading emergency doctor may want to inform the organizational medical leader about the number of victims. The tool will automatically create an appropriate service request from this. This request will then be matched with the available service offers. Such offers might include services totally unrelated to the task at hand, e.g. a service scheduling an ambulance, but also a number of services more or less suitable to achieve the given goal, e.g. the service offer above, but also a service that creates a wireless link, a service that ensures that the information is transmitted by a messenger etc. Unfortunately, requests that look very similar from the semantic service description and matchmaking point of view, may require the usage of very different services in order to meet compliance requirements. For instance, if the leading emergency doctor wants to communicate just the number of victims, she may be able to use a different service (e.g., a volunteer messenger or a wireless transmission) than when she also wants to communicate personal information about those victims.

In the next section we will describe in more detail how the association between internal controls and process tasks or services can be done.

\section{Compliance in Emergency Management}

As described above, our current matchmaker is able to take functional and nonfunctional aspects reflected in the service offer and request descriptions into account to find the best matching services. The matchmaker, however, is not able to take into account rules and regulations that are defined elsewhere. Here, we propose to integrate tools developed in the context of compliance management in BPM. In this section, we describe how concepts known there can be applied to our domain. 
Let us consider the following example: Article $\S 10$ of the Medical Association's professional code of conduct in Germany states that the leading medical doctor evaluates the operational picture: progress of the care of the patients on-site, the condition of the patients and the evacuation of the patients on-site. According to $\S 163$ StPO (German Code on Criminal Procedure), he is prohibited from spreading this information to other organisations (e.g., police). Unfortunately, not all emergency staff manages those requirements carefully or is even fully aware of them. Therefore, we suggest to start with formalizing the compliance requirements. We propose the use of formalisation as in [4] to facilitate the extension of service descriptions. In [4], the work examined the semantics of the contract conditions with reference to behavioural expressions which then they referred to as deontic constraints. Deontic constraints express what parties to the contact are required to perform (obligations), what they are allowed to do (permissions), or what they are not allowed to do (prohibitions). As a result, we derive compliance rules in form of machine readable statements/rules as follows: (1) Service Obligation - what must the services be able to deliver or produce? (2) Service Prohibition - what must the service not deliver or produce? (3) Service Delegation - what is permissible for the service to deliver or produce or substitute in terms of its output?

Consider our example about transmitting a message about affected persons from the leading emergency doctor to the organizational medical leader. An organizational medical leader leads the subordinate forces and is responsible for the best possible care of the patients. The subordinate forces forward information to the organizational medical leader. Thus, transmitting information about affected persons to the organizational medical leader from the leading emergency doctor is allowed and in this case the warranted connection is via an e-mail service ( $\$ 203$ StGB (The German Criminal Code) “...Transmitting patient's data ... via telephone or radio connection is basically forbidden"). These rules can be regarded as compliance rules. For instance, the Service Obligation part could capture the following requirements:

- Triage documentation, i.e., the estimation of victims injury in order to determine the priority of patients treatments based on the severity of their condition;

- Medical documentation of patients;

- Organization of ambulance movement according to the destination of patients.

In the Service Prohibition part will be a statement on what the service must not produce; regarding our example:

- Transmitting patient's data to other organizations (e.g., police, fire brigades) and

- Transmitting patient's data via telephone or radio connection.

The Service Delegation part will contain statements on what is acceptable to be substituted and describes the tasks to be executed in Compliance Management component. In our case these substitutes can be:

- Assignment of an identification number of patient (without supporting any medical documentation e.g. patient diagnose) to police in accordance with official call;

- Providing e-mail services as option for transmitting patient's data between leading emergency doctor and organizational medical leader. 
In the following, we show the examples of formalization for part of emergency services rules involving patient's data protection namely $\mathrm{R} 1, \mathrm{R} 2$, and $\mathrm{R} 3$. R1 represents compliance rule formalization specifying leading emergency doctor (LNA) has the obligation $(O)$ to safe keep patient's data. In the event of violation $(\otimes)$, LNA is obligated $(O)$ to pay fine. Another formalization, R2 shows that LNA is prohibited $(O \neg)$ to send patient's data to Non-Medical Agencies (NMA). We complete this with service delegation in R3 where LNA is permitted $(P)$ to send patient data through e-mail to Organizational Medical Leader (OrgL).

- R1 (Service Obligation): PatientData, $O_{\mathrm{LNA}}$ Safekeep $\otimes O_{\mathrm{LNA}}$ PayFine.

- R2 (Service Prohibition): PatientData, $O \neg$ LNA SendtoNonMedicalAgencies.

- R3 (Service Delegation): PatientData, $P_{\text {LNA }}$ SendEmailtoOrgL.

The examples have demonstrated how we use compliance rules to capture requirements in the emergency management domain. While semantic services provide the basis for dynamic process composition, in many settings, it is inadequate to find a functionally correct process and at the same time adhere to surroundings constraints (legal, etc.). Using compliance rules (in addition to the service descriptions) can provide us with the ability to dynamically allocate resources, execute services/substitutes, etc. in critical situation without violating required policies and guidelines.

\section{Solution Architecture}

In this section we present the overall architecture for representation of compliance requirements for emergency management.

In order to assist such dynamic environments, a system has to combine expertise about dynamically creating processes and expertise to generically describe and enforce compliance to reach the next level towards automatic process management. Figure 3 shows the interplay between the AI planner, the service component and the compliance management component: While the planning component will create processes that will solve given tasks, the service component will find concrete resources that are needed to execute these services. Service offers and service requests are not initially designed to fit together but are matched at run time by the service oriented software. In order to match a service request with a service offer, we have a service repository. It manages a list of all currently available services. To keep the repository up to date, service providers have the task to announce just offered services as well as services that are not available anymore. Service requests have the possibility to search in the repository for services providing certain functionality. In order to ensure that only service that match functionally and do not violate any rules and regulations, a compliance management component is needed. This component allows an on-the-fly compliance check and ensures that existing rules are fulfilled.

Emergency organizations typically involve a collection of business process tasks performed to accomplish the offered service. Considering that, we employ the use of ontological alignment and process model enrichment proposed by [5] in our solution. According to [5], process model enrichment will provide a better understanding of the interaction between process model and compliance rules (through improved visualisation of controls on the process model). Therefore, we start our compliance 


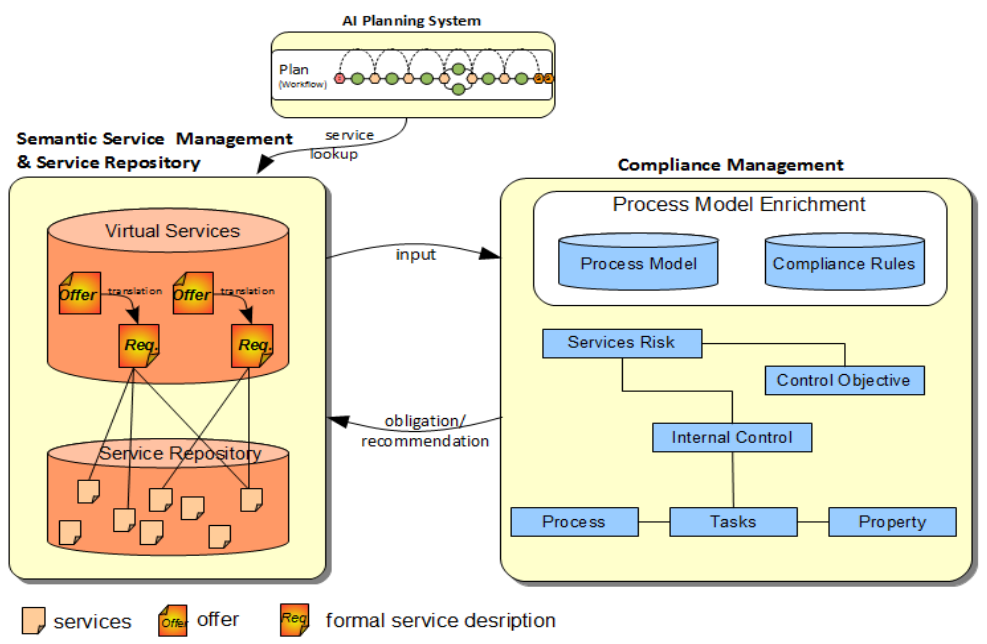

Fig. 3. System Architecture

management by identifying the associated compliance rules (regulation/standard) for a particular business process. Then, we proceed with the identification of risks for a particular service with regard to (violation of) compliance rules for the offered services. This will facilitate the identification of compliance obligation associated with a particular service or so called control objectives.

Following this, specific internal controls (specific activities/checks etc. that will be undertaken to fulfill a control objective) are designed and associated with the requisite control objectives and identified services risk. The fulfillment of control objectives will ensure the service process is free from the determined risks, or at least operates at a mitigated level of risk. To describe the relationship between internal controls and tasks, we observe that there are several controls that are applicable to a particular task. For each task there are a number of properties which represent the instance of a particular task. For instance, in emergency services the property may represent particular emergency staff roles or particular condition and/or time when a particular service is performed. By completing the ontological alignment and the process model enrichment, the emergency services and processes are explicitly associated with internal controls and hence with compliance obligations (or control objectives). This will facilitate the creation of low risk or risk free processes for a particular service adhering to compliance rules.

\section{Related Work}

In the last twenty years, many projects dealt with support in MCI and other emergency scenarios and many used a similar combination of technologies as SpeedUp does. Examples include SHARE [6], CoSAR-TS [7], MobiKat [8] and the Emergency Management System [9]. However, despite clear indications for the need $[10,11]$, to the best of our knowledge, none of these projects aims to provide sufficient artefacts 
with respect to compliance management for different types of rescue forces for managing MCI scenarios.

Efforts towards supporting the modeling of business process to meet compliance requirements have been established by a number of prior research works. In particular, [5] introduces an approach that provides the capability to capture compliance requirements through a generic requirements modeling framework. In their approach [5] also introduce the use of Formal Contract Language (FCL) as formalism to represent the declarative nature of compliance requirements. The formalization of process models is also supported by [12] that present formalism for annotated process models for the purpose of compliance checking. Another work by [13] provides an interesting method for integrating risks in business processes. The proposed technique for "risk-aware" business process models is developed for EPCs (Event Process Chains) using an extended notation. Similarly [14] present a logical language PENELOPE, that provides the ability to verify temporal constraints arising from compliance requirements on affected business processes. Distinct from the above works, the contribution of this paper has been on firstly providing an overall methodology for a model driven approach to business process compliance, and secondly on a structured technique for process model enrichment based on formal modeling of control objectives. Another significant research also exists on the modeling of control flow in business processes, particularly in the use of patterns to identify commonly used constructs [www.workflowpatterns.com]. On a similar note, [15] provide temporal rule patterns for regulatory policies, although the objective of this work is to facilitate event monitoring rather than the usage of the patterns for support of design time activities.

The most closely related work with regard to discovery is the WSMO-MX Matchmaker [16]. WSMO-MX is a hybrid matchmaker for WSML services that borrows the graph-matching approach from the DSD Matchmaker, but combines it with other concepts developed within other matchmakers which the DSD Matchmaker is lacking. What distinguishes the DSD Matchmaker most from WSMO-MX, as from most other discovery approaches is DSD's concept of precise fine-grained preferences and ranking. Most matchers proposed for OWL-S (see e.g. [17] for a typical example) rely on the subsumption matching of inputs and outputs described above and do not take the effects of the service into account. The recent matcher proposed in [18] additionally matches service product and classification. In contrast, DSD's matching is purely state-based. This has two advantages: (1) compares whether a service provides the desired effect or not and (2) DSD can abstract from differing interface and find functionally matching services even if their interface differ.

For WSMO, a discovery mechanism that abstracts from the individual effect to a desired, more generic goal is proposed. In [19] the developers argue that this abstraction is necessary due to performance considerations. Likewise, OWL-S [20], e.g., inherits difficulties with reasoning on the instance level from the description logics it is based on. Tools for OWL-S thus tend to not use instance information. However, contrary to DSD both approaches do not differentiate between a description of an offered and a requested service and rely on ontologies and heavy-weight logics (like description logics or frame logics). Thus, when searching for a service in dynamic and unreliable environments, it would be difficult to describe what should happen in cases where, e. g., information is missing or not fully matching. 


\section{Conclusion}

In this paper we have presented a high level model for the representation of compliance requirements technologies which are essential for building up IT-support for operation managers in large-scale MCI scenarios. We described the components, their functionalities in the modeling and execution phases. In our current approach, a model for representing compliance requirements is build through the extension of service descriptions in form of machine readable statement/rule. Most parts of the architecture are still in development and need additional scientific investigations such as the usage of these extensions in the matching process and the role of these extensions at service execution time. Currently, we are integrating the building blocks of our solution into a common prototype which will then be tested in a real-life setting.

Acknowledgements. The work described here has been partially funded by the Federal Government's program Research for Civil Security (call "rescue and protection of people") by the German Federal Ministry of Education and Research (http://www.speedup.uni-jena.de) and German Academic Research Service (DAAD).

We would like to thank our SpeedUp project partners from the Intercultural Business Communication (IWK) department, University of Jena (Germany) especially Yeliz Yildirim-Krannig, Mareike Mähler, and Fabian Wucholt who have provided us with important insights into intra- and inter-organizational interaction and contents of communication in MCIs. We also would like to acknowledge Shazia Sadiq from School of Information Technology and Electrical Engineering, The University of Queensland for her invaluable input in this research.

\section{References}

1. Peter, H.: Die Leitstelle beim MANV. Stumpf + Kossendey Verlagsgesellschaft mbH (2001)

2. Kemper, H.: Führen und Leiten im Einsatz. Ecomed, 3. Auflage 2008 (2008)

3. Kuester, U., Koenig-Ries, B., Klein, M., Stern, M.: DIANE - A Matchmaking-Centered Framework for Automated Service Discovery, Composition, Binding and Invocation on the Web. Special Issue of IJEC (International Journal of Electronic Commerce) on Semantic Matchmaking and Retrieval (2007)

4. Governatori, G., Milosevic, Z., Sadiq, S., Orlowska, M.: On Compliance of Business Processes with Business Contracts Technical Report, School of Information Technology and Electrical Engineering, The University of Queensland (2006)

5. Sadiq, S., Governatori, G., Namiri, K.: Modeling Control Objectives for Business Process Compliance. In: Alonso, G., Dadam, P., Rosemann, M. (eds.) BPM 2007. LNCS, vol. 4714, Springer, Heidelberg (2007)

6. Konstantopoulos, S., Pottebaum, J., Schon, J., Schneider, D., Winkler, T., Paliouras, G., Koch, R.: Ontology-Based Rescue Operation Management, pp. 112-121 (2009)

7. Tate, A., Dalton, J., Bradshaw, J.M., Uszok, A.: Coalition Search and Rescue - Task Support: Intelligent Task Achieving Agents on the Semantic Web. Technical report, Artificial Intelligence Applications Institute The University of Edinburgh and Florida Institute for Human \& Machine Cognition, IHMC (2004) 
8. Danowski, K.: MobiKat - Integriertes System zur Untersttzung der Katastrophenbewältigung und der alltäglichen Gefahrenabwehr. Fraunhofer-Institut für Verkehrs- und Infrastruktursysteme IVI Infoblatt (2007)

9. Rausch, A., Niebuhr, D., Schindler, M., Herrling, D.: Emergency Management System. In: Proceedings of the International Conference on Pervasive Services 2009 (ICSP 2009), Clausthal University of Technology, Department of Informatics - Software Systems Engineering Research Group (2009)

10. Robillard, J., Sambrook, R.: USAF Emergency and Incident Management Systems: A Systematic Analysis of Functional Requirements (2008),

http: / / www.uccs.edu/ rsambroo//Research/EIM_REQS.pdf

11. Lanfranchi, V., Ireson, N.: User requirements for a collective intelligence emergency response system. In: BCS-HCI 2009: Proceedings of the 23rd British HCI Group Annual Conference on People and Computers, pp. 198-203. British Computer Society, Swinton (2009)

12. Weber, I., Governatori, G., Hoffmann, J.: Approximate Compliance Checking for Annotated Process Models. In: 1st International Workshop on Governance, Risk and Compliance - Applications in Information Systems (GRCIS 2008) Held in Conjunction with the CAiSE 2008 Conference, Montpellier, France (2008)

13. Padmanabhan, V., Governatori, G., Sadiq, S., Colomb, R., Rotolo, A.: Process Modeling: The Deontic Way. In: Stumptner, M., Hartmann, S., Kiyoki, Y. (eds.) Australia-Pacific Conference on Conceptual Modeling, CRPIT, vol. 53, pp. 75-84 (2006)

14. Goedertier, S., Vanthienen, S.: Designing Compliant Business Processes with Obligations and Permissions. In: Eder, J., Dustdar, S., et al. (eds.) BPM Workshops 2006. LNCS, vol. 4103, pp. 5-14. Springer, Heidelberg (2006)

15. Giblin, C., Muller, S., Pfitzmann, B.: From Regulatory Policies to Event Monitoring Rules: Towards Model Driven Compliance Automation. IBM Research Report. Zurich Research Laboratory (October 2006)

16. Kaufer, F., Klusch, M.: WSMO-MX: A Logic Programming Based Hybrid Service Matchmaker. In: Proceedings of the 4th IEEE European Conference on Web Services (ECOWS 2006), Zürich, Switzerland (December 2006)

17. Paolucci, M., Kawamura, T., Payne, T.R., Sycara, K.P.: Semantic Matching of Web Services Capabilities. In: Horrocks, I., Hendler, J.A. (eds.) ISWC 2002. LNCS, vol. 2342, pp. 333-347. Springer, Heidelberg (2002)

18. Srinivasan, N., Paolucci, M., Sycara, K.: Semantic Web Service Discovery in the OWL-S IDE. In: Proceedings of the 39th Annual Hawaii International Conference on System Sciences, vol. 06. IEEE Computer Society, Los Alamitos (2006)

19. Keller, U., Lara, R., Polleres, A., Toma, I., Kifer, M., Fensel, M.: WSMO Web Service Discovery - WSML Working Draft 1211 2004. Technical Report, DERI (2004)

20. Burstein, M.H., Hobbs, J.R., Lassila, O., Martin, D.L., McDermott, D.V., Mcllraith, S.A., Narayanan, S., Paolucci, M., Payne, T.R., Sycara, K.P.: DAML-S: Web Service Description for the Semantic Web. In: Horrocks, I., Hendler, J. (eds.) ISWC 2002. LNCS, vol. 2342, p. 348. Springer, Heidelberg (2002) 